\title{
Poster Abstract: Measurements on the Efficiency of Overlapping Channels
}

\author{
Ozlem Durmaz Incel, Sape Mullender, Pierre Jansen, Stefan Dulman \\ University of Twente, The Netherlands \\ \{durmazo, sape, jansen, dulman\}@ cs.utwente.nl
}

\section{Introduction}

Wireless Sensor Networks (WSN) comprise a large number of embedded sensor devices with wireless communication capabilities. With the growing interest, soon WSN will be deployed everywhere in large numbers. The typical bandwidth offered by WSN is usually very limited (e.g., 50Kbps). Demand will be higher for the limited bandwidth in a dense deployment. This will result in a competitive communication environment and there will be less chance to access the wireless medium due to higher contention. Our aim is to use multiple channels to solve the problem of the contention in such a dense environment. Multiple simultaneous transmissions can take place on different channels without disturbing each other which leads to lower contention, fewer collisions and retransmissions.

The main focus of previous work on multi-frequency MAC protocols is how to utilize "non-overlapping" channels to increase the throughput of the network where the channels are assumed to be perfectly orthogonal. However, in practice channel orthogonality depends on factors like transmission power, distance between transmitters, etc. Arunesh et al. [2] propose the usage of overlapping channels with sufficient spatial distances to improve the application performance. In a previous work [1], we also show that there is a high correlation between spatial distance and the required channel distance to avoid interference between the simultaneous transmissions. The motivation of these studies is systematic usage of overlapping channels in the same spatial domain to support "non-interfering" simultaneous transmissions.

In this paper, we take a further step towards the systematic usage of overlapping channels and show that while having an acceptable level of interference we can improve the spectral efficiency. In the WSN domain, links are usually classified as good with $85 \%$ reception rate or more. These links are useful for communication since the packet loss can be handled by retransmissions. Accordingly, $15 \%$ or less interference (we define the interference level as the packet loss rate at the receivers) is acceptable in the WSN domain.

We use a typical radio platform for WSN to experiment the interference that occur among simultaneous transmissions on different channels. Although it can be argued that the results are specific to the example setting and platform, we show practical results on channel orthogonality and how we can adapt this orthogonality to utilize the spectrum usage and improve the network capacity. Throughput and throughput per-channel spacing $(t p-c s)$ are used as metrics. We show that one can adjust the channel spacing according to the level of interference tolerated by the application.

\section{Experiments}

The "Ambient $\mu$ Node" sensor node platform is used during the experiments. The platform is equipped with a single-chip radio transceiver that can operate on the $868 / 915 \mathrm{MHz}$ ISM band with a 50kbps data rate. An onboard dipole antenna is integrated. The radio frequency of the platform is adjustable. It provides 512 channels with $200 \mathrm{kHz}$ channel width.

Tests are performed in a large office. In the experiments, there are three different roles of the nodes: transmitter, receiver and jammer. The transmitter sends out packets with sequence numbers (256 packets for each run) and the receiver operates on the same frequency as the transmitter and maintains a $\log$ of the received packets. At the end of the tests, the data from the logger of a receiver is downloaded to be further analyzed. The jammer is also a transmitter whose operating frequency is adjusted to a different channel at each run. The jammer sends packets simultaneously with the transmitter, which allows us to observe the packet loss due to the interference between parallel transmissions on different channels.

In the experiments we use 2 jammers, 1 transmitter and 2 receivers. We place the nodes on a line topology: the transmitter and jammers are located next to each other, that is within a range of $10 \mathrm{~cm}, 10$ meters away 


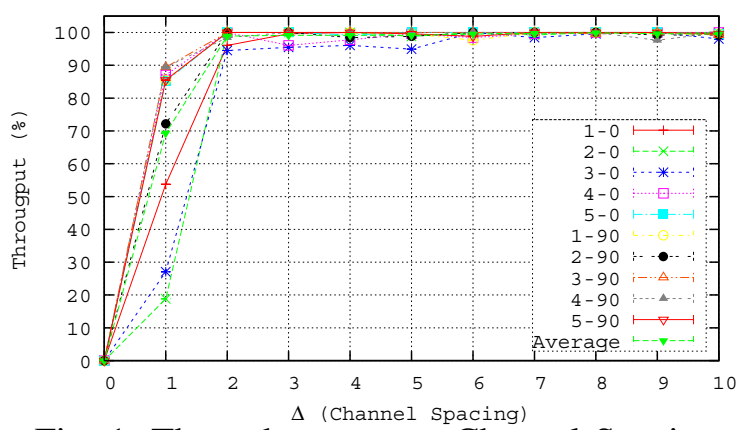

Fig. 1: Throughput versus Channel Spacing

from the receivers. We use 2 different receivers to investigate whether different antenna orientations impact the throughput. The transmitter and the receivers are tuned to the same channel $(873.6 \mathrm{MHz})$ while the jammers are alternating the operating channel on a different frequency at each run. For instance, when the transmitter is transmitting on channel $\mathrm{x}$, one of the jammers uses the channel $\mathrm{x}-\mathrm{y}$, and the other jammer uses $\mathrm{x}+\mathrm{y}$.

Figure 1 shows the results. The results for the receiver whose antenna is aligned with the antenna of transmitter are represented with a "-0" notation in the figure. The results for the other receiver with an orientation of 90 degrees are represented with a "-90" notation. The xaxis shows $\Delta$, the channel spacing between the jammer's operating frequency and a receiver's operating frequency (note that channel spacing is $200 \mathrm{KHz}$ between adjacent channels) whereas the y-axis shows the throughput (packet delivery rate) at a receiver. For the channel spacings not shown, the throughput is found to be $\cong 100 \%$. Different lines on the figure show the results taken at different times to show the stability of links over time (each line shows the averaged results of the experiments taken on the same day, different lines are for different days, 1 till 5). For different orientations of receivers, the results are similar with different levels of throughput. However, the same amount of channel spacing is required to avoid interference. The required channel spacing should be at least 3 with this setting of the nodes to have none-interfering simultaneous transmissions in the same spatial domain ( $\cong 100 \%$ throughput).

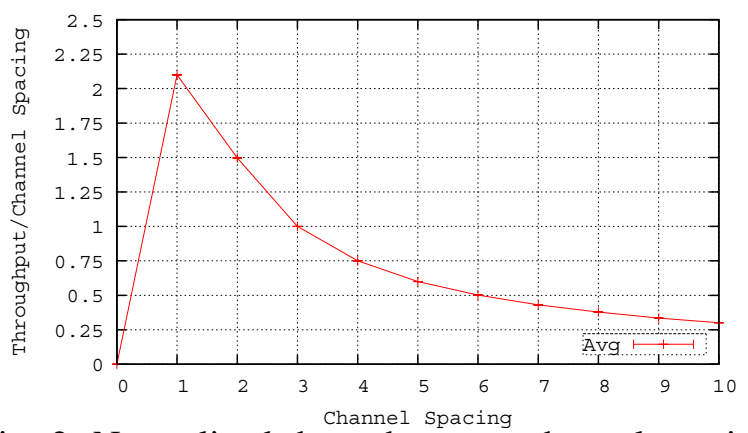

Fig. 2: Normalized throughput per-channel spacing
To represent the results in terms of $t p-c s$, we normalize them according to a baseline. Baseline is defined as maintaining zero packet loss with minimum $\Delta$. For example, if the distance between channels is $\Delta \mathrm{Hz}$ and $100 \%$ of throughput represents $\mathrm{T}$, then the baseline performance is $\mathrm{T} / \Delta$ (bits/s/Hz). If we narrow the gap to $\Delta / 2 \mathrm{~Hz}$ spacing and if the throughput drops by $15 \%$, we have $(T * 0.85) /(\Delta / 2)=1.7 T / \Delta$ (bits/s/Hz), which is better than the baseline. Figure 2 shows the results in terms of tp-cs. When the channel spacing is 3 , the average throughput is above $99 \%$, there's almost no interference and this is the baseline. We take the $t p$ cs as $1(\mathrm{~T} / \Delta)$ for a channel spacing of 3 . We compute the normalized throughput for each channel spacing, accordingly.

According to the results, to optimally utilize the spectrum and increase the spectral efficiency, it is not necessary to avoid interference completely. A channel spacing of 1 (Figure 2) gives the most optimal results in terms of the new metric.On the other hand, the average throughput is around $70 \%$ (Figure 1) with a channel spacing of 1 . This may not be acceptable for some applications. In that case, a channel spacing of 2 with $97 \%$ average throughput has better optimal results than a channel spacing of 3 with none interference. The metric of $t p-c s$ can be used to optimize the spectrum usage by keeping the interference level at an acceptable rate. According to the application requirements, one can adjust the channel spacing by the level of acceptable interference. Similarly, an acceptable level of interference can be determined by choosing an efficient channel spacing to improve the spectral efficiency and network capacity.

\section{Conclusions}

We have investigated the radio interference behavior of a multi-channel WSN system with a typical radio platform. We have analyzed the results in terms of throughput and throughput per-channel spacing. The experimental results show that wecan adjust the channel spacing with respect to the level of tolerated interference according to the application requirements. This gives us the possibility of simultaneous usage of as many as channels possible according to the permitted interference.

\section{References}

[1] O. D. Incel, S. Dulman, P. Jansen, and S. Mullender. Multi-channel interference measurements forwireless sensor networks. In Proceedings of LCN, pages 694-701, 2006.

[2] A. Mishra, V. Shrivastava, S. Banerjee, and W. Arbaugh. Partially overlapped channels not considered harmful. SIGMETRICS Perform. Eval. Rev., 34(1):63-74, 2006. 